\title{
EL GÉNERO ORMOSIA JACKS. (LEGUMINOSAE, PAPILIONOIDEAE) EN EL ESTADO DE GUERRERO, MÉXICO
}

\author{
RAMIro CRUZ-DurÁN ${ }^{1,2}$ Y JAIME JiMÉNEZ-RAMíREZ \\ ${ }^{1}$ Universidad Nacional Autónoma de México, Facultad de Ciencias, Departamento \\ de Biología Comparada, Ciudad Universitaria, Delegación Coyoacán, 04510 \\ México, D.F., México. \\ ${ }^{2}$ Autor para la correspondencia: ramcrudur@yahoo.com
}

\section{RESUMEN}

Se registra por primera vez la presencia del género Ormosia para el estado de Guerrero, México, con dos especies: Ormosia carinata y O. oaxacana (Leguminosae, Papilionoideae, tribe Sophoreae). Se incluyen las descripciones de ambas, datos referentes a los ejemplares, una clave dicotómica para diferenciarlas, y la ilustración de $O$. carinata. Ormosia carinata era conocida como elemento endémico de los estados de Veracruz y Oaxaca, en las regiones de Los Tuxtlas y de Uxpanapa.

Palabras clave: Guerrero, Leguminosae, México, Ormosia, Papilionoideae, Sophoreae.

\section{ABSTRACT}

Ormosia carinata and O. oaxacana (Leguminosae, Papilionoideae, tribe Sophoreae) are recorded for the first time from the state of Guerrero, Mexico. Descriptions for both species, lists of specimens studied, a dichotomous key to distinguish both taxa, and an illustration of $O$. carinata are provided. Oromosia carinata was formerly known only from the regions of Los Tuxtlas and Uxpanapa in Veracruz and Oaxaca.

Key words: Guerrero, Leguminosae, Mexico, Ormosia, Papilionoideae, Sophoreae.

En el trabajo de exploración botánica en el estado Guerrero se colectaron ejemplares de Ormosia carinata N. Zamora, conocida hasta ahora de las zonas de 
Los Tuxtlas (Veracruz) y de Uxpanapa (Oaxaca). Durante la revisión del Herbario Nacional de México (MEXU) y del Herbario de la Facultad de Ciencias (FCME) de la Universidad Nacional Autónoma de México, se encontraron también especímenes de $O$. oaxacana Rudd de Guerrero. La distribución previamente conocida de esta especie abarcaba a Oaxaca, Chiapas y Guatemala. Por ello, se decidió proveer descripciones y elaborar una clave para distinguirlas siguiendo el formato de la Flora de Guerrero (Diego et al., 1997).

Debido a las dificultades para colectar más plantas por la difícil situación política y social en esta entidad, hay relativamente poco material botánico disponible. En el caso de $O$. carinata la descripción se hizo con base en dos especímenes de Guerrero, mientras que los datos de semillas se tomaron de colectas de los estados de Veracruz y Oaxaca. Para O. oaxacana, de la que solo se cuenta para Guerrero con un ejemplar de herbario fértil en MEXU (con hojas, frutos y una semilla) y dos estériles en FCME, los caracteres correspondientes a partes florales se tomaron de ejemplares de la zona mesoamericana (estados de Oaxaca, Chiapas y Guatemala) y de lo referido por Rudd (1965).

Ormosia Jacks., Trans. Linn. Soc. London 10: 360. 1811.

Especie tipo: Ormosia coccinea (Aubl.) Jacks.

Árboles o arbustos; tallos en ocasiones con contrafuertes; corteza con anillos evidentes; ramillas glabras o ferrugíneo tomentosas. Hojas alternas, en ocasiones opuestas, particularmente en las partes apicales, imparipinnadas, con hasta 13 folíolos; estípulas deciduas; folíolos elípticos, oblongos a ligeramente obovados, opuestos o en ocasiones los basales alternos, base obtusa, cordata a ligeramente cuneada, margen aplanado o claramente ondulado, ápice obtuso o cuspidado, venación secundaria evidente, en ocasiones con venación terciaria escaleriforme, glabros, estrigosos o velutino ferrugíneos. Inflorescencias terminales o axilares, generalmente en panículas, en ocasiones en racimos. Flores blancas, de color lila, rojizas a purpúreas; cáliz campanulado, 5 lobado, los lóbulos claramente imbricados, los superiores casi unidos en su totalidad; pétalos 5, libres; estambres 10, libres, recurvados; pistilo con estilo fuertemente recurvado y estigma terminal u oblicuo, con 2 lóbulos. Frutos aplanados o globosos, coriáceos o leñosos, dehiscentes, rojos, amarillentos, pardos o negros, con 1 a 3(5) semillas, en ocasiones con septos entre las semillas; semillas elipsoides, globosas o lenticulares, rojas o negras, bicoloras (rojo con negro). 
Ormosia incluye alrededor de 130 especies con distribución desde el sur de México hasta Bolivia y Venezuela, Trinidad y Tobago, Brasil, Antillas, así como en las porciones tropicales de Asia, Indonesia, Australia y Oceanía (Rudd, 1965; Lewis, 2005).

El género Ormosia pertenece a la tribu Sophoreae, que se caracteriza por presentar inflorescencias en racimos o panículas, cáliz con lóbulos valvados o imbricados en botón, estambres libres, la parte superior del estilo glabra, frutos no articulados y semillas en ocasiones con arilo (Polhill y Raven, 1980). Ormosia se reconoce por sus hojas imparipinnadas, con folíolos opuestos a subopuestos, cáliz con lóbulos imbricados, estilo curvado, con estigma terminal o introrso en algunos casos bilobado y semillas bicoloras (rojo con negro) o a menudo rojas o negras, sin arilo (Rudd, 1965).

Debido a que son árboles o arbustos de gran talla, las colecciones resultan escasas y muchos ejemplares de herbario carecen de frutos. Asimismo, son pocos los tratamientos taxonómicos para el género; de éstos se pueden citar los de Standley (1920-1926) y Rudd $(1965,1981)$ para México, los de Dillon (1980) de Panamá, de Crowder (2001) en Nicaragua y de Zamora (2010) para Costa Rica.

Es importante resaltar que para el sureste de México se tienen reportadas a Ormosia carinata N. Zamora, O. isthmensis Standl., O. macrocalyx Ducke, O. oaxacana Rudd y $O$. schippii Pierce ex Standl. \& Steyerm., emend. Rudd; sin embargo, para Guerrero no se tenía registro del género.

Clave para las especies de Ormosia en el estado de Guerrero

1 Fruto sin carina o ala en el lado vexilar, sin septos que separan las semillas; folíolos completamente glabros, con margen aplanado; flores moradas a lila

O. oaxacana

1 Fruto con carina prominente, ala en el lado vexilar y septos que separan las semillas; folíolos con envés estrigoso, con margen ondulado; flores blancas

O. carinata

Oromosia carinata N. Zamora, Edinburgh J. Bot. 63(2-3): 183. 2006. Tipo: México. Veracruz, Municipio San Andrés Tuxtla: cerro Lázaro Cárdenas, Estación Biológica Los Tuxtlas, S. Sinaca \& F. Chigo 699 (Holotipo: K; Isotipos: CAS, MEXU!, MO!, NY). Fig. 1.

Árboles hasta $10 \mathrm{~m}$ de alto; ramillas pardo-velutinas; corteza lisa, con manchas pardo-verdosas o blancas, con lenticelas en líneas longitudinales; corteza inter- 
na amarilla; madera crema-amarillenta. Hojas 26 a 33(37) $\mathrm{cm}$ de largo, con (5)7 folíolos; estípulas deciduas; pecíolos (4.2)5.5 a $10.2 \mathrm{~cm}$ de largo, glabros; raquis glabro; peciólulos $1 \mathrm{~cm}$ de largo, glabros; folíolos (10.5)13 a $18 \mathrm{~cm}$ de largo y 4.5 a 7(9) $\mathrm{cm}$ de ancho (cuando secos adquieren tonos rosados), elípticos a obovados, base cuneada a obtusa, margen entero, ondulado, ápice ligeramente cuspidado, haz glabro, envés con escasos pelos estrigosos amarillos, que cuando maduran adquieren una tonalidad pajiza, verde-amarillento; con hasta 10 pares de venas secundarias, haz y envés con venación reticulada evidente. Inflorescencias hasta $20 \mathrm{~cm}$ de largo, en racimos axilares en las ramas terminales; raquis y pedúnculo velutinos, pardo-amarillentos; pedicelos 5 a $8 \mathrm{~mm}$ de largo, pardos, velutinos, con tricomas rojizos en el punto de unión con el raquis de la inflorescencia, brácteas y bracteólas caedizas. Flores $2 \mathrm{~cm}$ de largo; cáliz hasta $1 \mathrm{~cm}$ de largo, velutino, pardo-amarillento, con lóbulos $0.5 \mathrm{~cm}$ de largo; corola nívea; estandarte $16 \mathrm{~mm}$ de largo, con lámina $12 \mathrm{~mm}$ de largo y $16 \mathrm{a}$ $17 \mathrm{~mm}$ de ancho, ampliamente obovada, mácula verde-amarillenta en el centro, uña $5 \mathrm{~mm}$ de largo; alas 15 a $16 \mathrm{~mm}$ de largo, con lámina 11 a $12 \mathrm{~mm}$ de largo y 5 a 6 $\mathrm{mm}$ de ancho, oblongas, uña 3 a $4 \mathrm{~mm}$ de largo; pétalos de la quilla $15 \mathrm{~mm}$ de largo, con láminas $11 \mathrm{~mm}$ de largo y 6 a $7 \mathrm{~mm}$ de ancho, con tricomas pardos en la parte unida, base de color lavanda, uña $4 \mathrm{~mm}$ de largo; androceo 11 a $16 \mathrm{~mm}$ de largo; pistilo $15 \mathrm{~mm}$ de largo, ovario ca. $7 \mathrm{~mm}$ de largo, velutino, estilo ca. $8 \mathrm{~mm}$ de largo, ligeramente piloso en toda su longitud, recurvado cerca del ápice, estigma con el lóbulo distal curvo. Legumbres 5 a $7 \mathrm{~cm}$ de largo y 4.2 a $4.5 \mathrm{~cm}$ de ancho, elípticas, ligeramente aplanadas, en ocasiones globosas, amarillas, con 2 semillas separadas por un amplio septo, superficie lisa, lado vexilar con una giba o carina prominente hasta $0.5 \mathrm{~cm}$ de alto y ala hasta $1 \mathrm{~cm}$ de ancho. Semillas (1.4) 1.7 a $1.8 \mathrm{~cm}$ de largo y 1.1 a $1.3 \mathrm{~cm}$ de ancho, elípticas, rojas, hilo ca. $2 \mathrm{~mm}$ de largo (tomados de ejemplares del estado de Veracruz).

De información obtenida de los ejemplares de Veracruz depositados en MEXU, se sabe que la germinación es hipógea, en donde los dos primeros folíolos son simples y opuestos, y el tercero también es simple, pero alterno.

Ejemplares examinados: México. Guerrero. Municipio Iliatenco: desviación a Iliatenco, 1659'46.9" N, 98040'32.5" O, 22 abril 2012 (fl, fr (inmaduro)), J. Jiménez \& K. Vega 2012-153 (FCME); carretera a Iliatenco, 1659'47.3" N, 98²40'30.4" O, 17 marzo 2012 (fl, fr (viejo, sin semillas)), J. Jiménez \& K. Vega 2012-82 (FCME). Veracruz. Municipio Hidalgotitlán: aprox. $4 \mathrm{~km}$ por el camino al E del entronque de terracería La Laguna - Sarabia, 230 m s.n.m., 27 noviembre 1981 (fr), T. Wendt 
Cruz Durán y Jiménez Ramírez: Ormosia en el estado de Guerrero, México

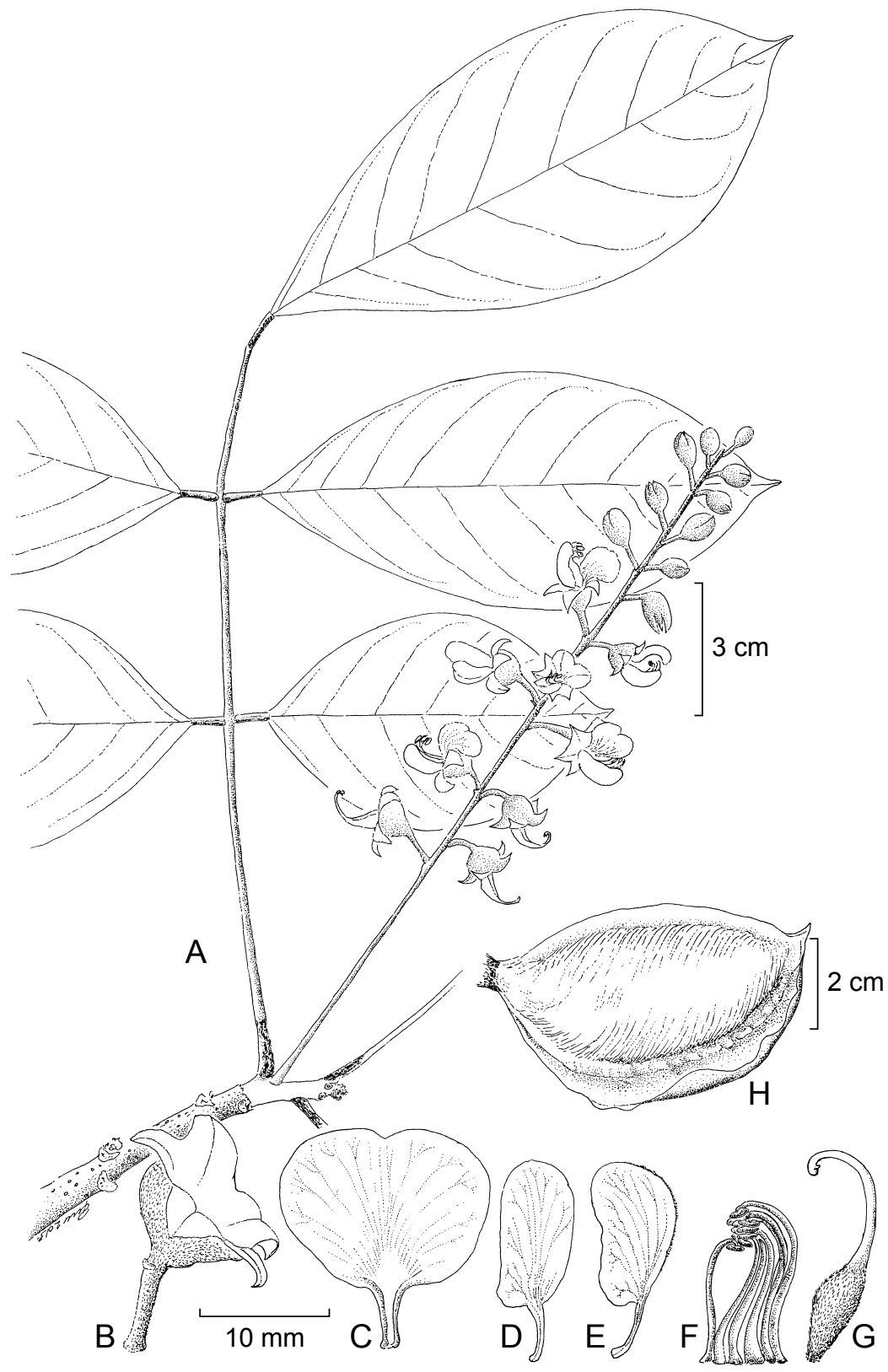

Fig. 1. Ormosia carinata N. Zamora. A. rama con flores en racimo; B. cáliz; C. estandarte; D. ala; E. pétalo de la quilla; F. androceo; G. pistilo; H. fruto. A, basado en Jiménez \& Vega 2013-153 (FCME); B-H, basados en Jiménez \& Vega 2012-82 (FCME). Ilustración de Ramiro Cruz Durán. 
et al. 3493 (MEXU); municipio Jesús Carranza: Lomas al $\mathrm{S}$ de Poblado 2, $3 \mathrm{~km}$ al $\mathrm{S}$ del entronque de la terracería La Laguna - Sarabia con el camino del $\mathrm{N}$ al poblado 2, 12 marzo 1984 (fl), T. Wendt et al. 4324 (MEXU; UAMIZ); $3 \mathrm{~km}$ al S del entronque de la terracería La Laguna - Boca del Monte, 250 m s.n.m., 30 mayo 1983 (fr), T. Wendt et al. 4174 (MEXU), 16 octubre 1983 (fr), T. Wendt \& I. Almaraz 4191 (MEXU); municipio San Andrés Tuxtla: Cerro Lázaro Cárdenas, Estación de Biología Tropical Los Tuxtlas, 500 m s.n.m., 22 mayo 1986 (fl), G. Ibarra \& S. Sinaca 2870 (MEXU); lote 69, Cerro Lázaro Cárdenas, Los Tuxtlas, 22 mayo 1989 (fl), G. Ibarra \& S. Sinaca 3386 (MEXU), 200 m s.n.m., 24 agosto 1989 (fr), G. Ibarra 3445 (MEXU); Estación de Biología Los Tuxtlas, 600 m s.n.m., 9 agosto 1985 (fr), S. Sinaca 187 (MEXU), 450 m s.n.m., 17 agosto 1985 (fr), S. Sinaca 208 (MEXU), 450 m s.n.m., 12 diciembre 1985 (fr), S. Sinaca 356, 357 (MEXU); camino a cerro Lázaro Cárdenas, 560 m s.n.m., 6 mayo 1986 (fl), S. Sinaca 556, 560, 700, 701, 702 (MEXU); cerro Lázaro Cárdenas, Estación de Biología Tropical Los Tuxtlas, 400 m s.n.m., 6 mayo 1986 (fl), S. Sinaca \& F. Chigo 699 (MEXU); camino a cerro Lázaro Cárdenas, 450 m s.n.m., 10 diciembre 1986 (fr), S. Sinaca 1141, 1143 (MEXU). Oaxaca. Municipio Matías Romero: $11 \mathrm{~km}$ al E de Aserradero Río Escondido, 177'16" N, 94³8'29" O, 250 m s.n.m., 14 abril 1986 (fl), H. Hernández \& V. Sánchez U-11 (MEXU).

Distribución: Guerrero, Oaxaca y Veracruz.

Altitud conocida en Guerrero: 1040 a $1094 \mathrm{~m}$.

Hábitat: bosque mesófilo de montaña; selva alta perennifolia en Veracruz y Oaxaca.

Fenología: florece y fructifica de marzo a abril.

Discusión: Por mucho tiempo se ha confundido esta planta con Ormosia panamensis Benth. ex Seem., conocida de Panamá, Nicaragua y Costa Rica. Sin embargo, Zamora (2006) describió $O$. carinata como una especie nueva de los estados de Veracruz y Oaxaca (en las zonas de Los Tuxtlas y Uxpanapa). En estas regiones crece en selva alta perennifolia asociada a Elaegia uxpanapensis, Dialium sp., Licania hypoleuca, Eschweilera sp., Byrsonima sp., Symphonia sp., Clethra sp. y Pouteria sp., en altitudes de 200 a 560(600) m, con floración en (marzo) mayo y fructificación de agosto a diciembre. En los ejemplares registrados para Guerrero las hojas y los folíolos son más grandes, las primeras de 26 a $33(37) \mathrm{cm}$ de largo, con 5-7 folíolos, de (10.5)13 a $18 \mathrm{~cm}$ de largo y de 4.5 a 7(9) $\mathrm{cm}$ de ancho, contra hojas de 15 a $27 \mathrm{~cm}$ de largo, con (5)7(9) folíolos, de 5.5 a 
$12.5 \mathrm{~cm}$ de largo y de 2 a $4 \mathrm{~cm}$ de ancho, en ejemplares de Veracruz y Oaxaca. En los últimos estados las plantas presentan mayor número de folíolos, y estos son de dimensiones menores.

Esta especie se parece a $O$. panamensis Benth. ex Seem., pues la última presenta frutos con superficie verrucosa y carentes de giba, sin embargo, O. carinata los tiene con superficie lisa y una giba prominente cercana al ala dorsal (carinal), esta de hasta $1 \mathrm{~cm}$ de alto. Asimismo, $O$. panamensis tiene folíolos con más pares de venas secundarias, hasta 13 , en tanto, O. carinata solo llega a presentar 10. En $O$. panamensis la flor es de color lavanda o blanca con el estandarte con mácula de color lila, mientras que $O$. carinata la corola es blanca y el estandarte presenta mácula verde.

Ormosia carinata también tiene cierta similitud con $O$. macrocalyx Ducke, pero difiere en la pubescencia y en la cantidad de folíolos. En $O$. macrocalyx se presentan generalmente 9 folíolos, rara vez 11 y estos son completamente glabros, mientras que en $O$. carinata el número común es 7 , excepcionalmente 9 , y tienen escasos pelos estrigosos en el envés.

Ormosia oaxacana Rudd, Bol. Soc. Bot. Méx. 41: 157. 1981. Tipo: México. Oaxaca, Finca Monte Cristo, cerro Espino, M. Sousa 7128 (Holotipo: MEXU!; Isotipos: MEXU!, MO!).

Árboles de 15 a 25 m de alto; corteza lisa con placas de líquenes encostrados. Hojas hasta $27.5 \mathrm{~cm}$ de largo, con 9-11 folíolos; estípulas deltado-lineares 1.7 a $3.5 \mathrm{~mm}$ de largo; pecíolos $6 \mathrm{~cm}$ de largo; raquis glabro; peciólulos $3 \mathrm{~mm}$ de largo, puberulentos con pelos pardos, con abundantes tricomas glandulares rojizos en el punto de unión con el raquis; folíolos 5.5 a $9(13.5) \mathrm{cm}$ de largo y 3 a $3.8(6.2) \mathrm{cm}$ de ancho, oblongos, los basales generalmente elípticos, base cuneada a obtusa, en ocasiones ligeramente cordata, margen aplanado, ápice obtuso a agudo, haz brillante, glabro, envés opaco, glabro, en algunos casos la vena media pelosa; con 11 a 13 pares de venas secundarias, medianamente realzadas en el envés, haz con venación de cuarto grado evidente. Inflorescencias 12 a 32(40) $\mathrm{cm}$ de largo, raquis, pedicelos y cáliz ferrugíneos. Flores 1.2 a $1.3 \mathrm{~cm}$ de largo, moradas o de color lila; pedicelos 3 a $4 \mathrm{~mm}$ de largo; bractéolas subuladas, 2.5 a $3 \mathrm{~mm}$ de largo; cáliz $10 \mathrm{~mm}$ de largo, lóbulo inferior hasta $5 \mathrm{~mm}$ de largo; estandarte orbicular, $9 \mathrm{~mm}$ de largo y $12 \mathrm{~mm}$ de ancho; alas $13 \mathrm{~mm}$ de largo y 5 $\mathrm{mm}$ de ancho; quilla $13 \mathrm{~mm}$ de largo y sus láminas $5 \mathrm{~mm}$ de ancho; androceo ca. $15 \mathrm{~mm}$ de largo; pistilo ca. $10 \mathrm{~mm}$ de largo. Legumbres 4.6 a $6 \mathrm{~cm}$ de largo y 2.5 
a $2.8 \mathrm{~cm}$ de ancho, rómbicas, con 1 semilla, superficie rugosa, con indumento estrigoso de pelos cortos pardos. Semillas $1.2 \mathrm{~cm}$ de largo, $1.2 \mathrm{~cm}$ de ancho y 1 $\mathrm{cm}$ de grosor, globosas, rojas.

Ejemplares examinados: México. Chiapas. Municipio Tila: colonia Kokijar, 1300 m s.n.m., 1 julio 1983 (fl), A. Méndez 6088 (MEXU). Guerrero. Municipio Atoyac de Álvarez: 27 km al NE de Atoyac de Álvarez, 935 m s.n.m., 28 marzo 1983 (fr), E. Martínez \& J. C. Soto 3738 (MEXU); municipio Petatlán: El Jilguero, $2.2 \mathrm{~km}$ al OSO, faldas de cerro El Jilguero, $17^{\circ} 35^{\prime} 49.5^{\prime \prime} \mathrm{N}, 101^{\circ} 3^{\prime} 26.8^{\prime \prime} \mathrm{O}, 1253$ m s.n.m., 4 mayo 2008 (estéril), F. Madariaga et al. 880 (FCME). Oaxaca. Dto. Miahuatlán, municipio San Jerónimo: $41 \mathrm{~km}$ al O de San Jerónimo Miahuatlán, $16^{\circ} 0^{\prime} \mathrm{N}, 97^{\circ} 1^{\prime}$ O, 1390 m s.n.m., 20 marzo 1988 (fl), R. Torres 11921 (MEXU). Dto. Pochutla, municipio Pluma Hidalgo: cerro Espino, Finca Monte Cristo, cafetal Monte Cristo y Cafetal San Carlos, 24 febrero 1988 (fl), A. Campos \& J. Reyes 1475 (MEXU); $100 \mathrm{~m}$ antes de llegar a Finca Monte Cristo, 920 m s.n.m., 5 julio 1977 (fr), M. Ricker 30 (MEXU); 850 m s.n.m., 26 junio 1982 (fr), $R$. Torres \& $R$. Cedillo 671 (MEXU); Finca Monte Cristo, cerro Espino, 850 m s.n.m., 7 febrero 1977 (fr), M. Sousa 7128 (MEXU, MO). Guatemala. Alta Verapaz, municipio Cobán: Chico'sa, 8 km al SO de Cobán, 15²6' N, 90²7' O, 22 julio 1988 (fl), $P$. Tenorio et al. 14672 (MEXU).

Distribución: México (Chiapas, Guerrero, Oaxaca), Guatemala.

Altitud en Guerrero: 925-1253 m.

Hábitat: selva mediana perennifolia, bosque mesófilo de montaña. En suelo amarillo arcilloso.

Fenología: fructifica en marzo.

Nombre común: palo verde, zumpancle (Oaxaca).

Usos: como sombra para cafetales.

Discusión: Ormosia oaxacana es similar a O. macrocalyx Ducke; sin embargo, la primera tiene folíolos largamente elípticos a completamente oblongos, con mayor número de pares de venas secundarias, de 10 hasta 15 o 16. Además, en esta especie en el punto de unión de los peciólulos con el raquis se presentan abundantes tricomas glandulares rojizos, en tanto $O$. macrocalyx presenta folíolos elípticos a ovados, con 5 a 9 pares de venas secundarias y no presenta pelos glandulares. Así mismo, O. oaxacana tiene germinación hipógea y las plántulas presentan el primer par de hojas opuestas y unifolioladas. 


\section{AGRADECIMIENTOS}

A José Antonio Hernández, Laboratorio de Microcine, Facultad de Ciencias de la Universidad Nacional Autónoma de México por la digitalización de la figura. A la Dirección General de Asuntos del Personal Académico de la Universidad Nacional Autónoma de México por el apoyo económico a través del Programa PAPIIT IN-214112 de Investigación e Innovación Tecnológica. A las autoridades del Herbario Nacional de México (MEXU) por las facilidades otorgadas para consultar su colección.

\section{LITERATURA CITADA}

Crowder, Ch. 2001. Ormosia Jacks. In: Stevens, W. D., C. Ulloa, A. Pool y D. Cutaia (eds.). Flora de Nicaragua. Monogr. Syst. Bot. Missouri Bot. Gard. 85(2): 1039-1040.

Diego, N., Fonseca, R. M. y E. Velázquez. 1997. Presentación, guía para los autores y normas editoriales para Flora de Guerrero y Estudios Florísticos de Guerrero. Las Presas de Ciencias, Facultad de Ciencias, Universidad Nacional Autónoma de México. México, D.F., México. 15 pp.

Dillon, M. 1980. Ormosia. In: Woodson, R. y R. Schery (eds.). Flora of Panama, Family 83. Leguminosae. Ann. Missouri Bot. Gard. 67(3): 737-743.

Lewis, G. P. 2005. Tribe Sophoreae. In: Lewis, G., B. Schrire, B. Mackinder y M. Lock (eds.). Legumes of the world. Royal Botanic Gardens, Kew. Kew, UK. pp. 227-248.

Polhill, R. M. 1981. Tribe 2. Sophoreae. In: Polhill, R. M. y P. H. Raven (eds.). Advances in legume systematics. Part I. Royal Botanic Gardens, Kew. Kew, UK. pp. 213-230.

Rudd, V. E. 1965. The American species of Ormosia (Leguminosae). Contr. U.S. Natl. Herb. 32: 279-384.

Rudd, V. E. 1981. Ormosia (Leguminosae) in Mexico, including a new species from Oaxaca. Bol. Soc. Bot. Méx. 41: 153-159.

Standley, P. C. 1920-1926. Trees and shrubs of Mexico. Cont. U.S. Natl. Herb. 23: 1-1721.

Zamora, N. 2006. Two new species of Ormosia (Leguminosae-Papilionoideae, Sophoreae) from Mesoamerica. Edinburgh J. Bot. 63(2/3): 183-190.

Zamora, N. 2010. Fabaceae. In: Hammel, B. E., M. H. Grayum, C. Herrera, y N. Zamora (eds.). Manual de plantas de Costa Rica. Monogr. Syst. Bot. Missouri Bot. Gard. 114(5): 659-663. 